\title{
OMITTED RAYS AND WEDGES OF FRACTIONAL CAUCHY TRANSFORMS
}

\author{
R. A. HIBSCHWEILER ${ }^{\unlhd}$ and T. H. MACGREGOR
}

(Received 16 April 2004; revised 15 February 2005)

Communicated by P. Fenton

\begin{abstract}
For $\alpha>0$ let $\mathscr{F}_{\alpha}$ denote the set of functions which can be expressed

$$
f(z)=\int_{|\zeta|=1} \frac{1}{(1-\bar{\zeta} z)^{\alpha}} d \mu(\zeta) \text { for }|z|<1,
$$

where $\mu$ is a complex-valued Borel measure on the unit circle. We show that if $f$ is an analytic function in $\Delta=\{z \in \mathbb{C}:|z|<1\}$ and there are two nonparallel rays in $\mathbb{C} \backslash f(\Delta)$ which do not meet, then $f \in \mathscr{F}_{\alpha}$ where $\alpha \pi$ denotes the largest of the two angles determined by the rays. Also if the range of a function analytic in $\Delta$ is contained in an angular wedge of opening $\alpha \pi$ and $1<\alpha<2$, then $f \in \mathscr{F}_{\alpha}$.
\end{abstract}

2000 Mathematics subject classification: primary 30E20; secondary $30 \mathrm{H} 05$.

\section{Introduction}

Let $\Delta=\{z \in \mathbb{C}:|z|<1\}$ and $\Lambda=\{z \in \mathbb{C}:|z|=1\}$, and let $\mathscr{M}$ denote the set of complex-valued Borel measures on $\Lambda$. For $\alpha>0$, let $\mathscr{F}_{\alpha}$ denote the set of functions $f: \Delta \rightarrow \mathbb{C}$ for which there exists $\mu \in \mathscr{M}$ such that

$$
f(z)=\int_{\Lambda} \frac{1}{(1-\bar{\zeta} z)^{\alpha}} d \mu(\zeta) \text { for }|z|<1 .
$$

The power function in (1) denotes the principal branch. Each function given by (1) is analytic in $\Delta$. For $f \in \mathscr{F}_{\alpha}$, let $\|f\|_{\mathscr{F}_{\alpha}}=\inf \|\mu\|$, where $\|\mu\|$ denotes the total variation of $\mu$, and $\mu$ varies over all measures in $\mathscr{M}$ for which (1) holds. This defines a norm on $\mathscr{F}_{\alpha}$, and $\mathscr{F}_{\alpha}$ is a Banach space with respect to this norm. The family $\mathscr{F}_{1}$

(C) 2006 Australian Mathematical Society 1446-7887/06 $\$ \mathrm{~A} 2.00+0.00$ 
was first studied by Havin [2]. The general class $\mathscr{F}_{\alpha}$, where $\alpha>0$, was introduced in [5] and has been studied extensively. In [6] a survey is given about these so-called fractional Cauchy transforms.

Several conditions on an analytic function $f$ are known to be sufficient to imply $f \in \mathscr{F}_{\alpha}$. Most of these conditions are analytic. Here we are concerned with geometric conditions. The Riesz-Herglotz formula provides information of this type. It implies that if $f: \Delta \rightarrow \mathbb{C}$ is analytic and $f(\Delta)$ is contained in a half-plane, then $f \in \mathscr{F}_{1}$. Another result of this kind was obtained by Bourdon and Cima in [1], namely, if $f: \Delta \rightarrow \mathbb{C}$ is analytic and there are two oppositely directed rays in $\mathbb{C} \backslash f(\Delta)$, then $f \in \mathscr{F}_{1}$.

This paper contains generalizations of the two results described above. We show that if $f(\Delta)$ is contained in an angular wedge with opening $\alpha \pi$ and $1<\alpha<2$, then $f \in \mathscr{F}_{\alpha}$. Also if there are two nonparallel rays in $\mathbb{C} \backslash f(\Delta)$ which do not meet and the angles at infinity between these two rays are $\alpha \pi$ and $\beta \pi$, then $f \in \mathscr{F}_{\gamma}$, where $\gamma=\max \{\alpha, \beta\}$.

If $f(\Delta)$ is contained in an angular wedge of opening less than $\pi$, then $f \in \mathscr{F}_{1}$, but $f$ need not belong to $\mathscr{F}_{\alpha}$ for any $\alpha, 0<\alpha<1$. This holds more generally for bounded analytic functions. To see this, let

$$
f(z)=\sum_{n=1}^{\infty} \frac{z^{2^{n}}}{n^{2}}, \quad|z|<1 .
$$

The function $f$ is analytic and bounded in $\Delta$. However $f \notin \mathscr{F}_{\alpha}$ when $0<\alpha<1$. This is because the Taylor coefficients of $f$ do not satisfy the condition $a_{n}=O\left(n^{\alpha-1}\right)$, which is necessary for membership in $\mathscr{F}_{\alpha}$.

Finally we mention that if $f: \Delta \rightarrow \mathbb{C}$ is analytic and $\mathbb{C} \backslash f(\Delta)$ contains a ray, then $f \in \mathscr{F}_{2}[5$, Theorem 5].

\section{Preliminaries}

This section contains lemmas which will be used to prove the main results. The first two lemmas are in [5, Lemma 1]. Lemma 2.3 is in [3, Theorem 2]. Lemma 2.4 is known but we give a proof.

LEMMA 2.1. For every $\alpha>0, f \in \mathscr{F}_{\alpha}$ if and only if $f^{\prime} \in \mathscr{F}_{\alpha+1}$.

LEMMA 2.2. If $0<\alpha<\beta$, then $\mathscr{F}_{\alpha} \subset \mathscr{F}_{\beta}$.

LEMma 2.3. If $\alpha \geq 1, f \in \mathscr{F}_{\alpha}$ and the function $\varphi: \Delta \rightarrow \Delta$ is analytic, then the composition $f \circ \varphi \in \mathscr{F}_{\alpha}$. 
LEMMA 2.4. Suppose that $f: \Delta \rightarrow \mathbb{C}$ is analytic and $f^{\prime}$ belongs to Hardy space $H^{1}$. Then $f \in \mathscr{F}_{\alpha}$ for every $\alpha>0$.

ProOF. Suppose that $f^{\prime} \in H^{1}$ and let $g=f^{\prime}$. Let $\zeta=e^{i \theta}$. Then

$$
G(\zeta) \equiv \lim _{r \rightarrow 1-} g(r \zeta)
$$

exists for almost all $\theta$ in $[-\pi, \pi]$ and $G\left(e^{i \theta}\right) \in L^{1}([-\pi, \pi])$. Also $g$ is represented by the Cauchy formula

$$
g(z)=\frac{1}{2 \pi i} \int_{\Lambda} \frac{G(\zeta)}{\zeta-z} d \zeta, \quad|z|<1 .
$$

Equation (2) yields (1), where $d \mu(\zeta)=(G(\zeta) / 2 \pi i \zeta) d \zeta$ and hence $g=f^{\prime} \in \mathscr{F}_{1}$. By Lemma 2.2, $f^{\prime} \in \mathscr{F}_{\alpha}$ for $\alpha>1$. Lemma 2.1 implies that $f \in \mathscr{F}_{\alpha}$ for all $\alpha>0$.

LEMMA 2.5. Suppose that the function $g$ is analytic in a neighbourhood of $\Delta$. Let $N$ be a positive integer and suppose that $\left|\zeta_{n}\right|=1, \alpha_{n}>0$ for $n=1,2, \ldots, N$, and $\zeta_{n} \neq \zeta_{m}$ for $n \neq m$. Let

$$
f(z)=\frac{g(z)}{\prod_{n=1}^{N}\left(z-\zeta_{n}\right)^{\alpha_{n}}}, \quad|z|<1 .
$$

Then $f \in \mathscr{F}_{\alpha}$, where $\alpha=\max \left\{\alpha_{n}: 1 \leq n \leq N\right\}$.

Proof. We give the proof for the case $N=2$. A similar argument can be given for other values of $N$.

Suppose that $|\zeta|=|\sigma|=1, \zeta \neq \sigma, \beta>0$, and $\gamma>0$. Suppose that the function $g$ is analytic in a neighborhood of $\Delta$ and let

$$
f(z)=\frac{g(z)}{(z-\zeta)^{\beta}(z-\sigma)^{\gamma}}, \quad|z|<1 .
$$

We shall show that $f \in \mathscr{F}_{\alpha}$, where $\alpha=\max \{\beta, \gamma\}$.

The function $z \mapsto g(z) /(z-\sigma)^{\gamma}$ is analytic at $z=\zeta$, and hence

$$
\frac{g(z)}{(z-\sigma)^{\gamma}}=\sum_{m=0}^{\infty} a_{m}(z-\zeta)^{m},
$$

for $z$ in some neighbourhood of $\zeta$. Let $p$ be the least integer such that $p \geq \beta$ and let $s=p-\beta$. Then

$$
f(z)=\sum_{m=0}^{p-1} \frac{a_{m}}{(z-\zeta)^{\beta-m}}+(z-\zeta)^{s} h(z),
$$


where the function $h$ is analytic in some neighbourhood of $\zeta$. Suppose that $\beta$ is not an integer. Then

$$
\frac{d}{d z}\left[(z-\zeta)^{s} h(z)\right]=(z-\zeta)^{s} h^{\prime}(z)+s(z-\zeta)^{s-1} h(z) .
$$

Since $(z-\zeta)^{s}$ is bounded in $\bar{\Delta} \backslash\{\zeta\}$, this implies that there is a positive constant $A$ such that

$$
\left|\frac{d}{d z}\left[(z-\zeta)^{s} h(z)\right]\right| \leq A|z-\zeta|^{s-1},
$$

for $z \in \bar{\Delta}, z$ near $\zeta$, and $z \neq \zeta$. Likewise if $\gamma$ is not an integer, $q$ is the least integer such that $q \geq \gamma$ and $t=q-\gamma$, then

$$
f(z)=\sum_{m=0}^{q-1} \frac{b_{m}}{(z-\sigma)^{\gamma-m}}+(z-\sigma)^{t} k(z),
$$

where $k$ is a function analytic in some neighbourhood of $\sigma$ and $b_{m}(m=0,1, \ldots, q-1)$ are suitable constants. Thus

$$
\left|\frac{d}{d z}\left[(z-\sigma)^{\prime} k(z)\right]\right| \leq B|z-\sigma|^{t-1}
$$

for $z \in \bar{\Delta}, z$ near $\sigma$, and $z \neq \sigma$, where $B$ is a positive constant.

For $z \in \bar{\Delta} \backslash\{\zeta, \sigma\}$, let

$$
r(z)=f(z)-\sum_{m=0}^{p-1} \frac{a_{m}}{(z-\zeta)^{\beta-m}}-\sum_{m=0}^{q-1} \frac{b_{m}}{(z-\sigma)^{\gamma-m}} .
$$

The relations (5), (6) and (9) imply that there is a constant $C$ such that

$$
\left|r^{\prime}(z)\right| \leq C|z-\zeta|^{s-1},
$$

for $z \in \bar{\Delta}, z$ near $\zeta$, and $z \neq \zeta$. Likewise (7)-(9) imply that

$$
\left|r^{\prime}(z)\right| \leq D|z-\sigma|^{t-1}
$$

for $z \in \bar{\Delta}, z$ near $\sigma$, and $z \neq \sigma$, where $D$ is a positive constant.

The function $z \mapsto(z-\tau)^{u}$ belongs to $H^{1}$ when $|\tau|=1$ and $u>-1$. Hence the inequalities (10) and (11) and the fact that $r^{\prime}$ is analytic in $\bar{\Delta} \backslash\{\zeta, \sigma\}$ imply that $r^{\prime} \in H^{1}$. This proves that $r^{\prime} \in H^{1}$ when $\beta$ and $\gamma$ are not integers. A similar argument shows that $r^{\prime} \in H^{1}$ when only one of the numbers $\beta$ and $\gamma$ is not an integer. If both 
$\beta$ and $\gamma$ are integers then $r=0$. Therefore, in general, $r^{\prime} \in H^{1}$. Lemma 2.4 yields $r \in \mathscr{F}_{\delta}$ for every $\delta>0$.

Equation (9) gives

$$
f=f_{1}+f_{2}+r
$$

where

$$
f_{1}(z)=\sum_{m=0}^{p-1} \frac{a_{m}}{(z-\zeta)^{\beta-m}} \quad \text { and } \quad f_{2}(z)=\sum_{m=0}^{q-1} \frac{b_{m}}{(z-\sigma)^{\gamma-m}} .
$$

The function $z \mapsto 1 /(z-\zeta)^{\delta}$ belongs to $\mathscr{F}_{\beta}$ when $0<\delta \leq \beta$ and hence $f_{1} \in \mathscr{F}_{\beta}$. Likewise $f_{1} \in \mathscr{F}_{\gamma}$. Lemma 2.2 yields $f_{1} \in \mathscr{F}_{\alpha}$ and $f_{2} \in \mathscr{F}_{\alpha}$. Since $r \in \mathscr{F}_{\alpha}$, (12) implies that $f \in \mathscr{F}_{\alpha}$.

Lemma 2.5 contrasts with the following result obtained in [5, Lemma 1].

THEOREM 2.6. If $f \in \mathscr{F}_{\alpha}$ and $g \in \mathscr{F}_{\beta}$ then $f \cdot g \in \mathscr{F}_{\alpha+\beta}$.

Since the function $g$ in Lemma 2.5 is analytic in $\bar{\Delta}, g$ is a multiplier of $\mathscr{F}_{\delta}$ for every $\delta>0$ [4, Theorem 3.5]. This fact and Theorem 2.6 imply that the function $f$ in (3) belongs to $\mathscr{F}_{\alpha^{\prime}}$, where $\alpha^{\prime}=\sum_{n=1}^{N} \alpha_{n}$. Lemma 2.5 is clearly an improvement of this result. The assumption that $\zeta_{n} \neq \zeta_{m}$ for $n \neq m$ is critical in Lemma 2.5. To see how this is reflected in our argument, suppose that the numbers $\zeta_{n}(n=1,2, \ldots, N)$ are distinct, $\zeta_{2} \rightarrow \zeta_{1}$, and the numbers $\alpha_{n}$ are fixed. Suppose that $\alpha=\max \left\{\alpha_{1}, \alpha_{2}, \ldots, \alpha_{N}\right\}$. Then the norm $\|f\|_{\mathscr{F}_{\alpha}}$ of the corresponding function in (3) goes to infinity as $\zeta_{2} \rightarrow \zeta_{1}$.

\section{The main results}

Let $f$ be analytic in $\Delta$. In this section we give two geometric conditions on $f(\Delta)$ sufficient to imply that $f \in \mathscr{F}_{\alpha}$.

THEOREM 3.1. Suppose that the function $f: \Delta \rightarrow \mathbb{C}$ is analytic and let $\Phi=$ $\mathbb{C} \backslash f(\Delta)$.

(a) Suppose that $\Phi$ contains two nonparallel rays. Let $\alpha \pi$ and $\beta \pi$ denote the angles at $\infty$ between these two rays, where $\alpha \geq \beta$. If $\alpha<2$, then $f \in \mathscr{F}_{\alpha}$.

(b) If $\Phi$ contains a ray then $f \in \mathscr{F}_{2}$.

PROOF. First assume that $\Phi$ contains two nonparallel rays. Since $\alpha+\beta=2$, the assumptions imply that $1<\alpha<2$. We may assume that the rays do not intersect. 
Let $F$ denote a conformal mapping of $\Delta$ onto the complement of the two rays. The Schwarz-Christoffel formula gives

$$
F(z)=b \int_{0}^{z} \frac{\left(w-\zeta_{1}\right)\left(w-\zeta_{2}\right)}{\left(w-\zeta_{3}\right)^{\alpha+1}\left(w-\zeta_{4}\right)^{3-\alpha}} d w+c,
$$

where $\zeta_{1}, \zeta_{2}, \zeta_{3}$, and $\zeta_{4}$ are distinct points on $\Lambda$, and $b$ and $c$ are suitable complex numbers. Hence

$$
F^{\prime}(z)=\frac{g(z)}{\left(w-\zeta_{3}\right)^{\alpha+1}\left(w-\zeta_{4}\right)^{3-\alpha}},
$$

where $g$ is a quadratic polynomial. Since $3-\alpha<\alpha+1$, Lemma 2.5 yields $F^{\prime} \in \mathscr{F}_{\alpha+1}$. Hence Lemma 2.1 implies $F \in \mathscr{F}_{\alpha}$.

Since $f(\Delta) \subset F(\Delta)$ and $F$ is univalent, the function $\varphi=F^{-1} \circ f$ is analytic in $\Delta$ and maps $\Delta$ into $\Delta$. Since $F \in \mathscr{F}_{\alpha}$ and $\alpha>1$, Lemma 2.3 yields $f=F \circ \varphi \in \mathscr{F}_{\alpha}$. This proves the first assertion.

The second assertion can be proved in a similar way. The conformal mapping of $\Delta$ onto the complement of a ray has the form $F(z)=P(z) /(z-\zeta)^{2}$, where $P$ is a quadratic polynomial and $|\zeta|=1$. This yields $F \in \mathscr{F}_{2}$ and hence Lemma 2.3 yields $f \in \mathscr{F}_{2}$.

THEOREM 3.2. Suppose that $f$ is analytic in $\Delta$. If $f(\Delta)$ is contained in an angular wedge of opening $\alpha \pi$ and $1<\alpha<2$, then $f \in \mathscr{F}_{\alpha}$.

PROOF. The function $z \mapsto[(1+z) /(1-z)]^{\alpha}$ maps $\Delta$ one-to-one onto the wedge $\{w:|\arg w|<\alpha \pi / 2\}$. Hence there are complex numbers $b$ and $c$ such that the function defined by $F(z)=b[(1+z) /(1-z)]^{\alpha}+c$ maps $\Delta$ one-to-one onto the angular wedge containing $f(\Delta)$. The function $z \mapsto 1 /(1-z)^{\alpha}$ belongs to $\mathscr{F}_{\alpha}$. Let $h(z)=(1+z)^{\alpha}$. Since $\alpha>1, h^{\prime}$ is bounded. Thus $h^{\prime} \in H^{1}$ and it follows that $h$ is a multiplier of $\mathscr{F}_{\delta}$ for every $\delta>0\left[4\right.$, Theorem 3.5]. Therefore $F \in \mathscr{F}_{\alpha}$. Since $f(\Delta) \subset F(\Delta)$ and $F$ is univalent, we have $f=F \circ \varphi$, where the function $\varphi: \Delta \rightarrow \Delta$ is analytic. Since $F \in \mathscr{F}_{\alpha}$ and $\alpha>1$, Lemma 2.3 gives $f \in \mathscr{F}_{\alpha}$.

\section{References}

[1] P. Bourdon and J. A. Cima, 'On integrals of Cauchy-Stieltjes type', Houston J. Math. 14 (1988), $465-474$.

[2] V. P. Havin, 'On analytic functions representable by an integral of Cauchy-Stieltjes type', Vestnik Leningrad Univ. Ser. Mat. Meh. Astronom. 13 (1958), 66-79 (in Russian).

[3] R. A. Hibschweiler and T. H. MacGregor, 'Closure properties of families of Cauchy-Stieltjes transforms', Proc. Amer. Math. Soc. 105 (1989), 615-621. 
[4] _ 'Multipliers of families of Cauchy-Stieltjes transforms', Trans. Amer. Math. Soc. 331 (1992), $377-394$.

[5] T. H. MacGregor, 'Analytic and univalent functions with integral representations involving complex measures', Indiana Univ. Math. J. 36 (1987), 109-130.

[6] __ _ 'Fractional Cauchy transforms', J. Comput. Appl. Math. 105 (1999), 93-108.

University of New Hampshire

Department of Mathematics and Statistics

Durham, NH 03824

USA

e-mail: rah2@cisunix.unh.edu
Bowdoin College

Department of Mathematics

Brunswick, ME 04011

USA 